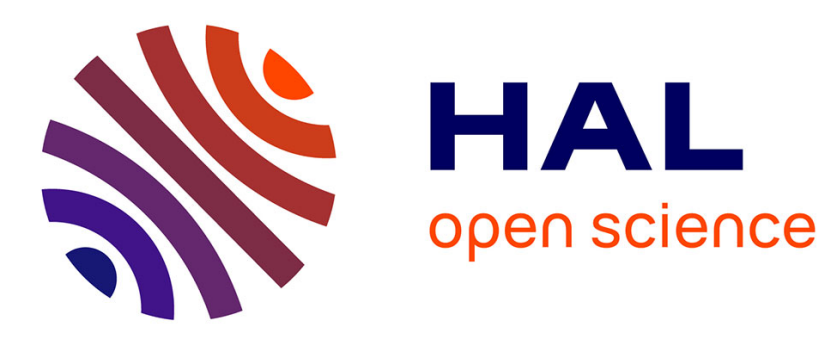

\title{
Les matériaux pour photopiles solaires
}

\author{
M. Rodot, M. Barbé, J. Dixmier
}

\section{To cite this version:}

M. Rodot, M. Barbé, J. Dixmier. Les matériaux pour photopiles solaires. Revue de Physique Appliquée, 1977, 12 (9), pp.1223-1235. 10.1051/rphysap:019770012090122300 . jpa-00244303

\section{HAL Id: jpa-00244303 https://hal.science/jpa-00244303}

Submitted on 1 Jan 1977

HAL is a multi-disciplinary open access archive for the deposit and dissemination of scientific research documents, whether they are published or not. The documents may come from teaching and research institutions in France or abroad, or from public or private research centers.
L'archive ouverte pluridisciplinaire HAL, est destinée au dépôt et à la diffusion de documents scientifiques de niveau recherche, publiés ou non, émanant des établissements d'enseignement et de recherche français ou étrangers, des laboratoires publics ou privés. 
Classification

Physics Abstracts

72.40

\title{
LES MATÉRIAUX POUR PHOTOPILES SOLAIRES
}

\author{
M. RODOT, M. BARBÉ et J. DIXMIER \\ Centre National de la Recherche Scientifique \\ Programme Interdisciplinaire de recherche sur le développement de l'énergie solaire (P. I. R. D. E. S.), \\ 75700 Paris, France
}

(Reçu le 15 février 1977, accepté le 25 mai 1977)

\begin{abstract}
Résumé. - Partant des objectifs technico-économiques de la recherche sur les photopiles solaires, on montre la nécessité de développer une deuxième filière n'utilisant pas les lingots de $\mathrm{Si}$ monocristallin. On discute les qualités que doit posséder le matériau choisi : possibilité d'un procédé d'élaboration économique, propriétés intrinsèques (absorption), longueur de diffusion des porteurs minoritaires, possibilité de construire une structure collectrice efficace, fiabilité, aptitude à l'usage sous concentration du rayonnement solaire. On conclut que trois filières paraissent particulièrement prometteuses : couches minces de Si polycristal ou amorphe, GaAs ou autre matériau III-V sous concentration, $\mathrm{CdTe}$ ou $\mathrm{Cu}_{2} \mathrm{~S}$ préparés par voie chimique à très bas prix.
\end{abstract}

\begin{abstract}
A discussion of the technico-economic goals of solar cell research shows the necessity of developing a second type of cells which do not use single crystal $\mathrm{Si}$ ingots. The qualities of possible materials are analyzed : possibility of a cheap procedure of elaboration, intrinsic properties (absorption), diffusion lengths of minority carriers, possibility of building an efficient collecting structure, reliability, capability of use under solar radiation concentration. It is concluded that three materials look specially promising : thin films of polycristalline or amorphous $\mathrm{Si}, \mathrm{GaAs}$ or other III-V materials under concentration, $\mathrm{CdTe}$ or $\mathrm{Cu}_{2} \mathrm{~S}$ prepared chemically at very low cost.
\end{abstract}

1. Introduction. - La conversion de l'énergie solaire est considérée parfois comme un gadget d'importance limitée, parfois comme la vraie réponse aux besoins de l'humanité au vingt et unième siècle. Cette incertitude la qualifie comme un sujet de recherche intéressant.

De même que le chauffage et la climatisation solaire, les centrales de conversion thermodynamique ou la production de fuel à partir de biomasse, la conversion photovoltaïque ne peut jouer un rôle important qu'à long terme. Bien qu'ils aient été développés il y a déjà vingt ans pour les applications spatiales, les générateurs photovoltaïques pour la production terrestre d'électricité représentent seulement une puissance totale installée de quelques centaines de kilowatts en 1977 et nul ne pense qu'ils puissent atteindre le niveau de puissance totale installée de plusieurs centaines de mégawatts avant une dizaine d'années; cependant la production actuelle de photopiles triple chaque année et un décollage massif de cette industrie est prévu par certains vers 1980 (aux Etats-Unis l'ERDA vise à produire $500 \mathrm{MW} / \mathrm{an}$ en $1986 \mathrm{au}$ prix de $2500 \mathrm{~F} / \mathrm{kW}$ installé).

Les prédictions peuvent être plus précises à court terme, car on peut définir des objectifs réalistes pour les années prochaines, en extrapolant les performances actuellement connues. Cette analyse $(\S 2 \mathrm{du}$ présent article) montrera que la technique actuelle, utilisant des tranches monocristallines de $\mathrm{Si}$, limite sévèrement les possibilités et qu'un des principaux problèmes à l'ordre du jour est de choisir de nouveaux matériaux semi-conducteurs conduisant à la fois à un bon rendement et à un bas prix.

Nous examinerons ensuite (§ 3) quelles qualités doit présenter un bon matériau photosensible. Ces qualités incluent, parmi d'autres : l'aptitude du matériau à être préparé en grandes surfaces économiques, certaines propriétés intrinsèques liées à l'absorption des photons, et extrinsèques liées à la recombinaison des porteurs de charge. Bien qu'il soit difficile à certains égards de considérer à part le matériau lui-même et la structure photovoltaïque, nous tenterons dans cet article de minimiser la discussion de la structure collectrice des porteurs de charges pour nous concentrer sur la discussion du matériau qui pose les problèmes les plus décisifs pour l'avenir des photopiles.

Nous étudierons ensuite, dans les paragraphes 4 à 9 , les points forts et les points faibles des différents matériaux. Nous montrerons que le Si lui-même n'est pas encore suffisamment bien caractérisé en dépit des nombreux travaux qui lui ont été consacrés. Les données sur les autres semi-conducteurs sont souvent très rares. C'est là une raison pour concentrer les recherches sur un petit nombre de matériaux parmi les plus prometteurs. D'ailleurs les matériaux ayant une chance de devenir 
compétitifs sont peu nombreux : ce sont surtout, outre le $\mathrm{Si}$ : GaAs, InP ou certains alliages III-V, CdTe ou $\mathrm{Cu}_{2} \mathrm{~S}$. Les tentatives de choisir d'autres matériaux plus ou moins exotiques sont susceptibles de conduire à des mises au point intéressantes, mais non d'ouvrir une voie nouvelle pratiquement valable. Mais l'étude des matériaux principaux doit être développée très finement pour permettre une bonne estimation de leurs possibilités.

Contrairement à une opinion fréquemment soutenue, nous ne conclurons pas à la supériorité certaine du Si à long terme. Les qualités du Si sont si grandes et sa technologie si avancée que son remplacement par d'autres matériaux paraît parfois peu probable. Cependant, pour des objectifs tels que des centrales de $1 \mathrm{MW}$ en site isolé, les nouvelles formes de $\mathrm{Si}$ actuellement étudiées (rubans ou couches polycristallines) sont en compétition avec GaAs ou CdTe par exemple. Aucun générateur n'a encore été construit avec l'un ou l'autre de ces matériaux.

2. But des recherches sur les photopiles solaires. Considérons deux objectifs d'inégale difficulté :

a) Alimenter des pompes à eau au Sahel. La compétitivité est assurée si l'eau pompée de $20 \mathrm{~m}$ de profondeur revient à moins de $0,15 \mathrm{~F} / \mathrm{m}^{3}$, ou encore si l'énergie électrique coûte moins de $1,50 \mathrm{~F} / \mathrm{kWh}$.

b) Construire une centrale de puissance en site isolé. En ordre de grandeur, ceci exige un coût de l'énergie inférieur à $0,50 \mathrm{~F} / \mathrm{kWh}$.

Les panneaux solaires classiques comportant des cellules constituées de tranches de Si mono-cristallin et utilisés sans concentration, valent $11000 \mathrm{~F} / \mathrm{m}^{2}$ ou $100000 \mathrm{~F} / \mathrm{kW}$ crête, d'où un prix de l'énergie de $15 \mathrm{~F} / \mathrm{kWh}$ : 10 fois trop pour l'application $a$ ), 30 fois trop pour l'application $b$ ).

On peut envisager d'atteindre l'objectif $a$ ) par des panneaux de cellules au Si classiques, car le prix de ces derniers devrait baisser d'un facteur 3 ou 4 d'ici 1980 (sans changement technique majeur) et l'emploi d'une certaine concentration conduit à un nouveau facteur favorable de 3 ou 4 au moins qui se cumule avec le précédent. Mais l'objectif $b$ ), plus ambitieux, semble être hors de portée de la technologie actuelle. Ceci résulte principalement du coût du matériau. Ce dernier est aujourd'hui de $4000 \mathrm{~F} / \mathrm{m}^{2}$, il devrait descendre au-dessous de $500 \mathrm{~F} / \mathrm{m}^{2}$ si on vise l'objectif $b$ ) : ceci ne peut être atteint dans le cadre de la technique actuelle et exige la mise au point d'une nouvelle génération de photopiles.

Examinons maintenant le rendement de conversion exigé des cellules solaires. Nous avons calculé le rendement minimum nécessaire pour atteindre un objectif de coût donné (Fig. 1). Ce calcul prend en compte les coûts vraisemblables des supports de cellules, de l'électrotechnique associée (incluant un stockage modeste), etc... On voit que, même avec un coût de panneaux aussi faible que $500 \mathrm{~F} / \mathrm{m}^{2}$, le rendement doit

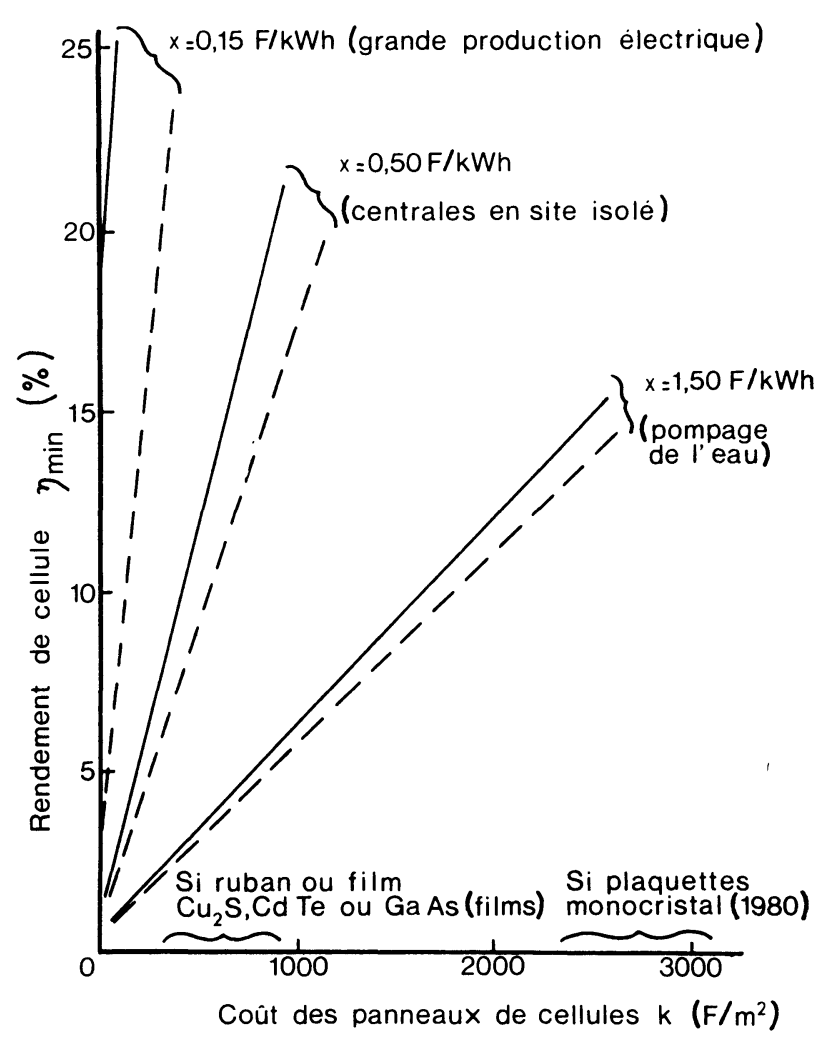

Fig. 1. - Rendement de conversion minimum $\eta_{\min }$ requis, pour un prix des panneaux de cellules $k$ donné, pour atteindre un prix de l'électricité $x$, sans concentration :- - sans stockage, avec stockage $(6 \mathrm{~h})$.

[Minimum cell efficiency $\left(\eta_{\min }\right)$ required, for a given panel cells cost $k$, to reach a given cost of electricity $x$, without use of concentration : - - - without storage, — with storage (6 hours).]

dépasser $10 \%$ pour remplir l'objectif $b$ ). On voit aussi qu'un éventuel objectif $c$ ), qui serait la production massive d'électricité, est pratiquement hors d'atteinte, surtout à cause du coût du stockage électrique.

La figure 1 a été tracée en l'absence de concentration du rayonnement solaire. Les conséquences négatives et positives de la concentration peuvent être évaluées, ce qui mène à un effet global positif illustré par la figure 2 dans le cas de cellules au Si refroidies naturellement. Cet effet est important (courbe A) pour les cellules solaires actuelles, moins important mais encore significatif pour les cellules en Si monocristal de 1980 ; le gain associé à la concentration sera encore plus faible pour les cellules très économiques de l'avenir (rubans ou couches minces) et probablement marginal pour la gamme de concentration de la figure 2. Cette conclusion pourra être modifiée pour les rapports de concentration plus importants autorisés par certains types de cellules (cf. $\S 9$ ).

Nous retiendrons en conclusion que de nouveaux types, améliorés, de cellules solaires devraient posséder les qualités suivantes : rendement supérieur à $10 \%$, coût de panneaux $<1000 \mathrm{~F} / \mathrm{m}^{2}$ ou aptitude à fonctionner sous haute concentration $(>300)$. 


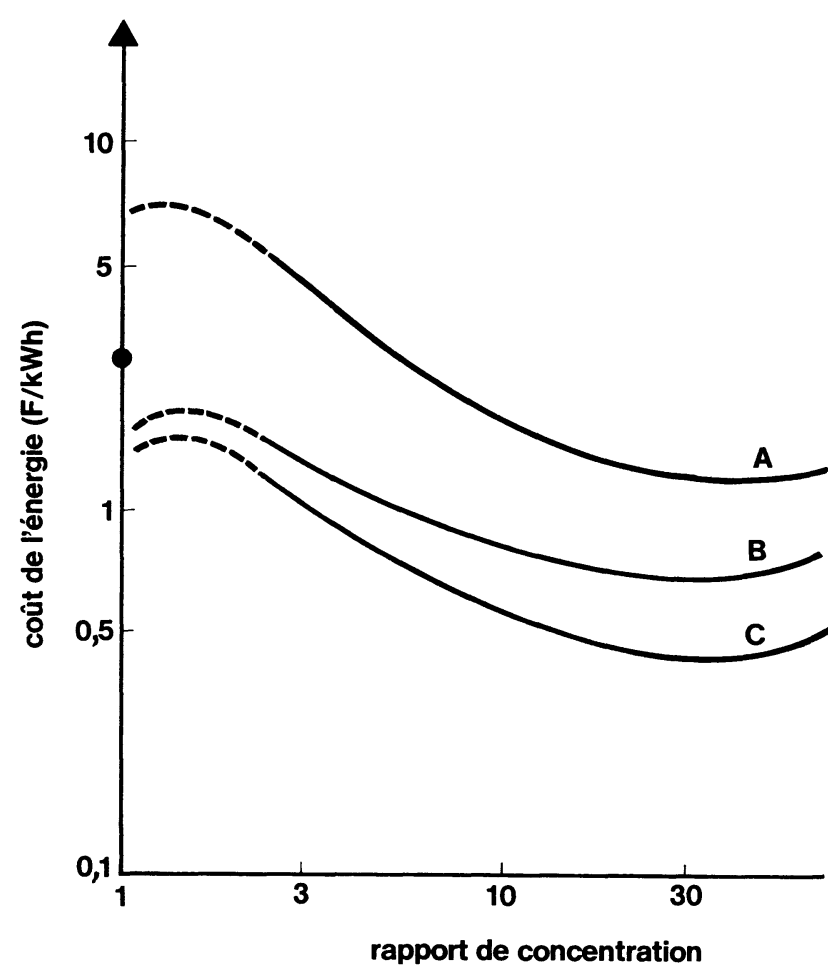

Fig. 2. - Coût de l'électricité produite par les cellules au Si sous concentration (refroidissement naturel à l'air). $\Delta$ panneaux actuels $\left(11000 \mathrm{~F} / \mathrm{m}^{2}\right)$ sans concentration ; Courbe $\mathrm{A}$ : panneaux actuels avec un concentrateur de coût $1000 \mathrm{~F} / \mathrm{m}^{2} ; 0$ panneaux de $1980\left(2500 \mathrm{~F} / \mathrm{m}^{2}\right)$ sans concentration ; Courbe B : panneaux de 1980 avec un concentrateur de coût $600 \mathrm{~F} / \mathrm{m}^{2}$; Courbe $C$ : panneaux de 1980 avec un concentrateur de coût $300 \mathrm{~F} / \mathrm{m}^{2}$.

[Cost of electricity produced by $\mathrm{Si}$ cells under concentration : $\Delta$ Present cells $\left(2.200 \$ / \mathrm{m}^{2}\right)$ without concentration. Curve A : present cells with concentrator cost $200 \$ / \mathrm{m}^{2}$; 1980 cells $\left(500 \$ / \mathrm{m}^{2}\right)$ without concentration; Curve B : 1980 cells with concentrator cost $120 \$ / \mathrm{m}^{2}$; Curve C : 1980 cells with concentrator cost $60 \$ / \mathrm{m}^{2}$.]

3. Qualités nécessaires d'un matériau pour photopiles. - a) La première exigence est la possibilité de préparer de grandes surfaces de matériau de bonne qualité électronique, de façon économique. Ceci implique une sélection à la fois des matériaux et des procédés d'élaboration. Le mot bonnes qualités électroniques indique que les propriétés ne doivent pas être dégradées notablement par rapport à celles des monocristaux les plus parfaits, compte tenu du rendement élevé qui est exigé.

b) L'optimisation de l'absorption des photons conduit à deux exigences bien connues. D'abord il existe une largeur de bande interdite $E_{\mathrm{g}}$ optimale, pour minimiser à la fois les pertes dues aux photons non absorbés $\left(h v<E_{\mathrm{g}}\right)$ et aux photons trop énergiques $\left(h v \gg E_{\mathrm{g}}\right.$ ). Pour des diodes idéales cet optimum se situe à $1,5 \mathrm{eV}$ ou à $1,9 \mathrm{eV}$ selon que la cellule fonctionne à $300 \mathrm{~K}$ ou à $500 \mathrm{~K}$, les rendements optimaux $\eta$ étant de $28 \%$ et $15 \%$ respectivement [1]. Pour des diodes non idéales, les valeurs de $E_{\mathrm{g}}$ et de $\eta$ décroissent quelque peu. Ensuite la nature des transitions bande-à- bande influe sur l'épaisseur de la cellule. Cette dernière doit être de l'ordre de la longueur d'absorption $1 / \alpha$ ( $\alpha=$ coefficient d'absorption moyen pour $h v>E_{\mathrm{g}}$ ), qui est beaucoup plus petite pour les transitions directes sans phonons ( 1 à $5 \mu \mathrm{m}$ pour $\mathrm{Cu}_{2} \mathrm{~S}, \mathrm{CdTe}$ ou $\mathrm{GaAs})$ que pour les transitions indirectes assistées par des phonons (50 à $100 \mu \mathrm{m}$ pour le $\mathrm{Si}$ ). Le cas de transitions directes autorise d'utiliser des cristaux de plus faible épaisseur, d'un autre côté il conduit à un accroissement de la mobilité et à une diminution de la durée de vie des porteurs minoritaires, deux facteurs qui influencent en sens contraire la longueur de diffusion et se compensent mutuellement.

c) La collection des porteurs de charge créés dans le matériau neutre est contrariée par les pertes par recombinaison, qui dépendent de la longueur de diffusion : $L=\sqrt{D \tau}$ (avec $D=k T \mu / q=$ coefficient de diffusion des porteurs). Pour éviter l'effet de ces pertes, la longueur de diffusion doit être du même ordre que la longueur d'absorption $1 / \alpha$. Un fort dopage du matériau photo-sensible décroît $L$, tandis qu'il a des avantages à d'autres points de vue (cf. $\$ \S 6$ et 7), de sorte qu'il existe un dopage optimal du matériau. D'autres pertes par recombinaison ont lieu à la surface du semiconducteur et sont caractérisées par une vitesse de recombinaison superficielle. Les porteurs de charge créés dans une zone de charge d'espace sont collectés sans perte, mais même dans le cas favorable du Si amorphe ces zones ne sont pas assez larges et des rendements intéressants ne sont accessibles que dans un matériau de forte longueur de diffusion [63, 64].

d) Une quatrième qualité requise est l'aptitude à construire une jonction collectrice efficace, et à prendre 1 ou 2 contacts ohmiques de faible résistance. La structure collectrice peut être une jonction p-n comme il est usuel pour le $\mathrm{Si}$, mais aussi une hétérojonction, une barrière métal-semi-conducteur ou une structure MIS.

e) De plus le matériau lui-même et la cellule complète doivent être fiables. Cette exigence est très sévère, car les données de la figure 1 ont été calculées en admettant une durée de vie des cellules égale à 20 ans, ce qui est aujourd'hui hautement optimiste.

$f$ ) Enfin l'aptitude à la concentration est aussi une propriété reliée à la fois au matériau et à la structure.

4. Procédés d'élaboration. - Les cellules solaires actuelles utilisent des tranches monocristallines de Si faites comme l'indique le tableau I. Ce procédé, qui fut conçu pour la microélectronique, est très cher; de plus il dépense une grande quantité d'énergie, de sorte que les cellules solaires actuelles sont amorties en énergie au bout de 12 ans seulement [3]. Cette difficulté doit (et peut) être résolue par les techniques décrites dans ce paragraphe.

Dans quelques années, il sera consommé autant de $\mathrm{Si}$ pour les photopiles que pour la microélectronique. Ce Si n'est pas tenu d'avoir la pureté requise pour cette 


\section{TABLEAU I}

Coût $d u$ Si (en francs 1975) [2]

[Cost of silicon [2]]

Forme initiale

Sable (pureté) $99 \%$

Si métallurgique

Chlorosilanes

$\mathrm{SiHCl}_{3}$

$\mathrm{SiHCl}_{3}$ pur

Si polycristal

Si monocristal
Traitement

1) Réduction par le coke au four à arc

2) Réaction avec $\mathrm{HCl}$ en lit fluidisé

3) Distill. fractionnée

4) Distill. fractionnée

5) Décomposition pyrolytique avec $\mathrm{H}_{2}$

6) Tirage Czochraliski

7) Découpage
Forme finale

Si métallurgique pureté

$98 \%$

Chlorosilanes

$\mathrm{Si} \mathrm{HCl}_{3}$

$\mathrm{SiHCl}_{3}$ pur. 99,999\%

Si polycristal (impuretés $\simeq 10^{-9}$ )

Si monocristal

Tranches $300 \mu \mathrm{m}$
F/kg Si

$\overline{0}, 025$

2,50

$\mathrm{F} / \mathrm{kW}$ crête 0,21

21

dernière application : comme le montre le tableau II, la plupart des impuretés peuvent être tolérées au niveau de 10 à $100 \mathrm{ppb}\left(1 \mathrm{ppb}=10^{-9}\right)$ alors que leur teneur actuelle est de $1 \mathrm{ppb}$; deux auteurs récents confirment

\section{TABLEaU II}

Performance de cellules au Si utilisant du Si impur [4]

[Performance of Si cells using impure Si [4]]

$\begin{array}{cccc}\begin{array}{c}\text { Impu- } \\ \text { retés }\end{array} & \begin{array}{c}\text { Limite de } \\ \text { solubilité } \\ \left(\mathrm{cm}^{-3}\right)\end{array} & \begin{array}{c}\text { Performance des cellules } \\ \text { (en \% } \\ \text { de celle du Si } \\ \text { ultra pur })\end{array} & \begin{array}{c}\text { par } \\ \text { addition de } \\ \left(10^{15} \mathrm{~cm}^{-3}\right)\end{array} \\ \mathrm{Cu} & 1 \times 10^{18} & - & - \\ \mathrm{Cr} & & 95 & 0,3-2 \\ \mathrm{Ti} & & 96 & 0,1 \\ \mathrm{Mn} & 4 \times 10^{16} & 88 & 0,01-1 \\ \mathrm{~V} & & 94 & 0,01-01 \\ \mathrm{Mg} & & 100 & 0,01-01 \\ \mathrm{Ni} & & 100 & 0,1-1 \\ \mathrm{Fe} & 3 \times 10^{16} & 97 & 0,01-01 \\ \mathrm{Al} & 2 \times 10^{19} & 98 & 0,01-0,7 \\ \mathrm{Au} & 3 \times 10^{16} & 90 & 20 \\ & & 90 & 0,7\end{array}$

que $\mathrm{Ti}, \mathrm{Zr}$ et $\mathrm{V}$ sont particulièrement dangereux et doivent être limités à 1 ou $2 \mathrm{ppb}$, mais que les limites tolérables atteignent environ $30 \mathrm{ppb}$ pour $\mathrm{Fe}$, plus de $100 \mathrm{ppb}$ pour $\mathrm{Mn}, \mathrm{Cr}, \mathrm{Cu}$ et près de $1000 \mathrm{ppb}$ pour Ni [5]. A la récente conférence de Baton Rouge il a été proposé [6] de court-circuiter les étapes 2 à 5 du tableau I, une pureté suffisante pouvant être obtenue par une réduction au four à arc effectuée avec soin et suivie d'un tirage Czochralski.

D'autre part on a étudié théoriquement [7] la diminution de rendement apportée par du Si polycristallin, lorsque les cristaux ont la forme de colonnes traversant la région active, et montré que le rendement du monocristal était conservé si le diamètre moyen des grains était 5 à 10 fois l'épaisseur de cette couche.

On voit sur la figure 3 qu'une taille de grain de $100 \mu \mathrm{m}$ est suffisante pour le $\mathrm{Si}$, et $10 \mu \mathrm{m}$ pour le GaAs. Cette conclusion a été récemment confirmée pour le $\mathrm{Si}[8]$. On avait déjà observé antérieurement que du Si

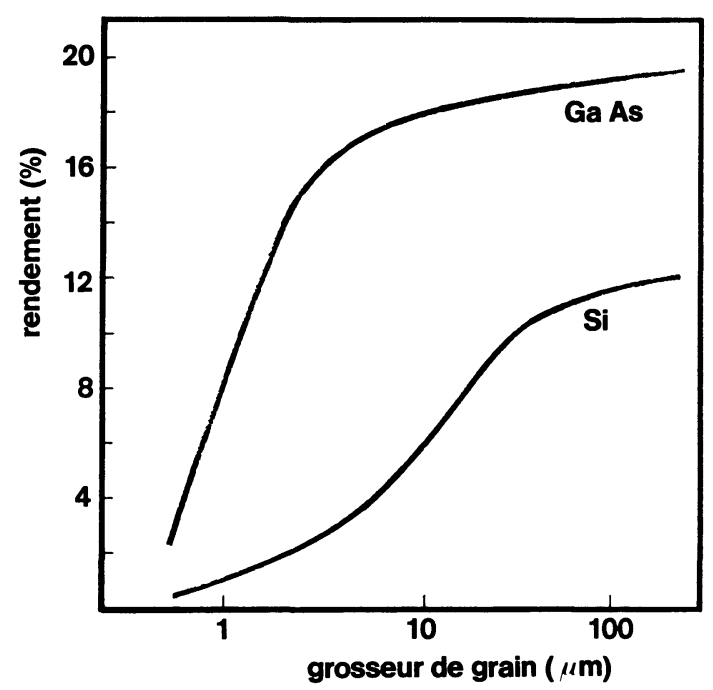

FIG. 3. - Effet de la grosseur de grain de Si et GaAs polycristallins sur le rendement de la photopile [7] (à partir de mesures de durée de vie, en supposant $V_{\text {oc }}$ indépendant des joints de grain).

[Effect of grain size of Si and GaAs polycrystal on photocell efficiency [7] (from lifetime measurements, assuming $V_{\mathrm{oc}}$ is independant of grain boundaries).]

polycristallin pouvait permettre de conserver des rendements de 6 à $9 \%$ [9].

Pour obtenir un abaissement substantiel du coût du $\mathrm{Si}$, on peut donc suivre une ou plusieurs des voies suivantes :

a) Gardant la technologie actuelle, accroître le diamètre des lingots, de 56 à 100 et même 150 à $200 \mathrm{~mm}$, et accroître l'efficacité du découpage (scies comportant par exemple 40 disques très fins). 
b) Simplifier le processus de purification $\mathrm{du}$ Si. Outre la technique déjà citée [6] on a proposé des procédés utilisant la dismutation $\mathrm{SiF}_{4}-\mathrm{SiF}_{2}$ [10] ou le traitement de $\mathrm{Si}$ par le $\mathrm{Zn} \mathrm{[11].}$

L'obtention d'un prix de $50 \mathrm{~F} / \mathrm{kg}$ dans quelques années semble possible, pour un $\mathrm{Si}$ de pureté modeste mais suffisante.

c) Eviter la découpe des monocristaux en obtenant directement le Si sous forme de rubans, par la technique des dendrites [12] ou celle de croissance en filière $(E F G=$ edge - defined film growth, ou méthode de Stepanov du nom de son premier inventeur). Cette dernière méthode conduit à des rubans d'épaisseur $100 \mu \mathrm{m}$, tirés à la vitesse de 1 à $4 \mathrm{~cm}$. min. ${ }^{-1}$, permettant un rendement de photopiles de $10 \%$ [13].

d) Utiliser du Si polycristallin. La technique citée ci-dessus [9] consiste à mouler du Si liquide, par exemple entre deux plaques de graphite, puis à découper le polycristal formé. On peut aussi utiliser la cristallisation du Si sur un ruban d'alumine [14] ou de papier graphité [15] trempé dans Si liquide ; cette méthode de trempage semble très prometteuse.

Alors que la croissance de monocristaux n'est guère possible à partir de Si impur, les voies $b$ ) et $d$ ) peuvent être combinées ensemble, sous réserve des effets de ségrégations aux joints de grain, encore mal connus.

Le tableau III résume le bilan des techniques de couches minces, non seulement pour le $\mathrm{Si}$, mais aussi pour d'autres matériaux potentiellement intéressants. Il est consacré aux cinq techniques qui devraient permettre d'atteindre les coûts les plus bas ; quelques coûts connus dans l'industrie du verre ou des métaux qui figurent dans la deuxième colonne peuvent être considérés comme des objectifs possibles.

La croissance en bain fondu est usuelle dans l'indus- trie des semi-conducteurs et devrait convenir à $\mathrm{Si}, \mathrm{CdTe}$ ou GaAs, sous réserve de vérification de la qualité cristalline en l'absence de conditions d'épitaxie. Ce procédé donne en général une vitesse de croissance élevée, il peut être appliqué en production continue ; ses désavantages sont son coût énergétique élevé (haute température) et la difficulté d'imaginer la production d'une cellule complète par ce procédé.

L'électrolyse est un procédé encore peu connu pour déposer des couches minces semi-conductrices (voir [17] pour le $\mathrm{Si}$ ).

La technique spray (pulvérisation chimique) consiste à atomiser des solutions liquides appropriées contenant les espèces réactives et à faire réagir ces dernières pour produire la couche mince désirée et d'autres espèces volatiles. Son application aux photopiles CdS$\mathrm{Cu}_{2} \mathrm{~S}$ [18] a donné d'intéressants résultats. Cette technique a un faible coût énergétique et une aptitude à produire de grandes surfaces, peut-être en continu ; cependant l'adhérence, l'homogénéité et la qualité électronique du film peuvent être difficiles à contrôler.

La «C. V. D. » (dépôt chimique en phase vapeur) est une technique bien connue pour $\mathrm{Si}$ et $\mathrm{GaAs}$. Son coût énergétique est modéré, le taux de croissance est bas (typiquement $10 \mu \mathrm{m} / \mathrm{h}$ ). Cette technique ne paraît pas se prêter à la production continue de grandes surfaces, ni à la production de cellules complètes par un seul procédé. Les propriétés électroniques des films déposés sur des supports économiques étaient satisfaisantes pour CdTe [19], mauvaises mais améliorables par recristallisation pour $\mathrm{Si}$ [16].

Sous la dernière rubrique, les techniques sous vide, nous considérons ensemble la sublimation et la pulvérisation cathodique. Ici le coût énergétique est élevé mais le taux de croissance l'est également ( $1 \mu \mathrm{m} / \mathrm{min}$.). De grandes surfaces peuvent être produites, mais non

\section{TABLEAU III}

Performances de divers procédés de croissance de couches minces

[Performances of various processes for thin film growing]

\begin{tabular}{|c|c|c|c|c|c|}
\hline Technique & $\begin{array}{l}\text { Prix limite } \\
\text { en tant que } \\
\text { procédé } \\
\text { industriel }\end{array}$ & $\mathrm{Si}$ & $\mathrm{Cu}_{2} \mathrm{~S} / \mathrm{CdS}$ & $\mathrm{CdTe}$ & GaAs \\
\hline Croissance en bain fondu & $?$ & $\begin{array}{c}{[14], \text { mullite }} \\
1450 \mathrm{C}, 4,5 \%\end{array}$ & $\overline{\text { non }}$ & faisable & faisable \\
\hline Electrolyse & $250 \mathrm{~F} / \mathrm{m}^{2}$ & $\begin{array}{c}{[17], \mathrm{Si}} \\
750 \mathrm{C}, ?\end{array}$ & $?$ & $?$ & $?$ \\
\hline Pulvérisation chimique & $250 \mathrm{~F} / \mathrm{m}^{2}$ & non & $\begin{array}{c}{[18], \text { verre }} \\
200 \mathrm{C}, 5 \%\end{array}$ & $?$ & non \\
\hline Dépôt chimique en phase vapeur & $?$ & $\begin{array}{l}\text { [16], Si métall. } \\
1000 \mathrm{C}, 2 \%\end{array}$ & $?$ & $\begin{array}{c}{[19], \text { Mo }} \\
500 \mathrm{C}, 6 \%\end{array}$ & faisable \\
\hline Procédés sous vide & $500 \mathrm{~F} / \mathrm{m}^{2}$ & non & $\begin{array}{r}{[20], \text { mylar }} \\
320 \text { C, } 7 \%\end{array}$ & faisable & non \\
\hline
\end{tabular}

$$
\text { Code : } \begin{aligned}
& \begin{array}{l}
\text { référence, substrat } \\
\text { température, rendement }
\end{array} \\
& \hline
\end{aligned}
$$




\section{TABLEAU IV}

Propriétés (à $300 \mathrm{~K}$ ) des matériaux pour photopiles

[Properties (at $300 \mathrm{~K}$ ) of solar cell materials]

\begin{tabular}{|c|c|c|c|c|c|c|c|c|}
\hline \multicolumn{2}{|l|}{$\begin{array}{c}\text { Structure } \\
\left({ }^{1}\right)\end{array}$} & $\begin{array}{l}\text { Ionicité } \\
{[22,23]}\end{array}$ & $\begin{array}{c}\text { Paramètre } \\
\text { de réseau } \\
\text { a }(\AA) \\
\left({ }^{1}\right)\end{array}$ & $\begin{array}{c}\text { Coefficient } \\
\text { de dilatation } \\
\left(10^{-6} \mathrm{deg}^{-1}\right) \\
{[24]}\end{array}$ & $\begin{array}{c}\text { Largeur } \\
\text { de bande } \\
\text { interdite } \\
E_{\mathrm{g}}(\mathrm{eV}) \\
\left.{ }_{(}^{2}\right)\end{array}$ & $\begin{array}{c}\text { Affinité } \\
\text { électronique } \\
(\mathrm{eV}) \\
{[24]}\end{array}$ & $\begin{array}{c}\mathrm{Mol} \\
\left(\mathrm{cm}^{2} \mathrm{~V}\right. \\
\text { électr. }\end{array}$ & $\begin{array}{l}\text { lité } \\
\text { trous }\end{array}$ \\
\hline $\mathrm{Si}$ & $\mathrm{D}$ & 0 & 5,431 & 2,33 & 1,11 (i) & 4,01 & 1350 & 480 \\
\hline InP & ZB & 0,421 & 5,869 & 4,5 & $1,27(\mathrm{~d})$ & 4,38 & 4500 & 100 \\
\hline GaAs & ZB & 0,310 & 5,654 & 5,8 & 1,43 (d) & 4,07 & 8000 & 300 \\
\hline $\mathrm{Ga}_{0,2} \mathrm{Al}_{0,8} \mathrm{Sb}$ & ZB & & 6,13 & 5 & 1,47 (i) & & & 400 \\
\hline $\mathrm{CdTe}$ & ZB & 0,675 & 6,477 & & 1,44 (d) & 4,28 & 700 & 65 \\
\hline $\mathrm{CdSe}$ & $\mathrm{H}$ & 0,699 & 4,3 & 4,8 & 1,7 (d) & 3,93 & 600 & \\
\hline $\mathrm{CdS}$ & $\mathbf{H}$ & 0,685 & 4,14 & 4,0 & 2,42 (d) & 4,5 & 340 & \\
\hline $\mathrm{CdSiAs}_{2}$ & $\mathrm{C}$ & 0,$54 ; 0,70$ & $5,884(1,849)$ & & $1,55(d)$ & & & 500 \\
\hline $\mathrm{CuInSe}_{2}$ & $\mathrm{C}$ & 0,$65 ; 0,57$ & $5,78 \quad(2,00)$ & & $1,04(d)$ & & 320 & 10 \\
\hline $\mathrm{CuInS}_{2}$ & $\mathrm{C}$ & 0,$68 ; 0,61$ & $5,52 \quad(2,00)$ & & 1,5 (d) & & 200 & 15 \\
\hline $\mathrm{Cu}_{2} \mathrm{~S}$ & $\mathrm{Ch}$ & & $\left({ }^{1}\right)$ & & 1,21 & 4,25 & & 24 \\
\hline InSe & $\mathbf{L}$ & & & & 1,24 & & 500 & \\
\hline
\end{tabular}

(1) $\mathrm{D}=$ diamant ; $\mathrm{ZB}=$ blende de zinc ; $\mathrm{H}=$ hexagonal (le paramètre à comparer avec celui des cristaux ZB pour l'épitaxie sur le plan (111), est $a \sqrt{2}$ ); $\mathrm{C}=$ chalcopyrite (rapport $c / a$ entre parenthèse); $\mathrm{Ch}=$ chalcocite (orthorhombique) : $a=11,86$; $b=27,32 ; c=13,49 ; L=$ structure en couche.

(2) (d) = bande interdite directe ; (i) = bande interdite indirecte.

en continu. Contrairement aux procédés précédents, le rapport du poids de film produit au poids de matière utilisée est très inférieur à 1 . Ce procédé est couramment utilisé pour $\mathrm{CdS} / \mathrm{Cu}_{2} \mathrm{~S}$. Un travail sur les alliages à base de $\mathrm{CdTe}$ [21] montre que probablement du bon CdTe peut également être obtenu par ce procédé.

Le tableau III montre combien le travail reste à faire sur les matériaux et les procédés mentionnés. Probablement des composés II-VI comme CdTe peuvent être obtenus avec des propriétés satisfaisantes par tous ces procédés ; la démonstration doit encore en être faite pour $\mathrm{GaAs}$ et $\mathrm{Si}$.

5. Propriétés intrinsèques des matériaux. - Le tableau IV montre quelques propriétés des matériaux les plus intéressants. Pour les séries de structure diamant-blende de zinc, la nature (directe ou indirecte) et la valeur de $E_{\mathrm{g}}$ sont interprétables par la théorie diélectrique de Phillips [22], qui peut donner quantitativement l'énergie de chaque branche de la bande de conduction (de symétrie $\Gamma$, $\mathrm{L}$ ou $\mathrm{X}$ ), par rapport au sommet $E_{\mathrm{v}}$ de la bande de valence (ainsi que l'affinité électronique $\chi$ ).

Dans une série d'alliages comme $\mathrm{Ga}_{1-x} \mathrm{Al}_{x} \mathrm{As}$ ou $\mathrm{Ga}_{1-x} \mathrm{Al}_{x} \mathrm{Sb}$, ces énergies varient continûment avec $x$ (Fig. 4) de sorte qu'il existe un point de croisement, séparant les alliages à bande interdite directe et indirecte.

Le coefficient d'absorption dépend de la longueur d'onde comme le montre la figure $5:$ le comportement différent des matériaux à bande interdite directe (CdTe, $\mathrm{GaAs}, \mathrm{InP})$ et indirecte $(\mathrm{Si}, \mathrm{GaP})$ est bien apparent ;

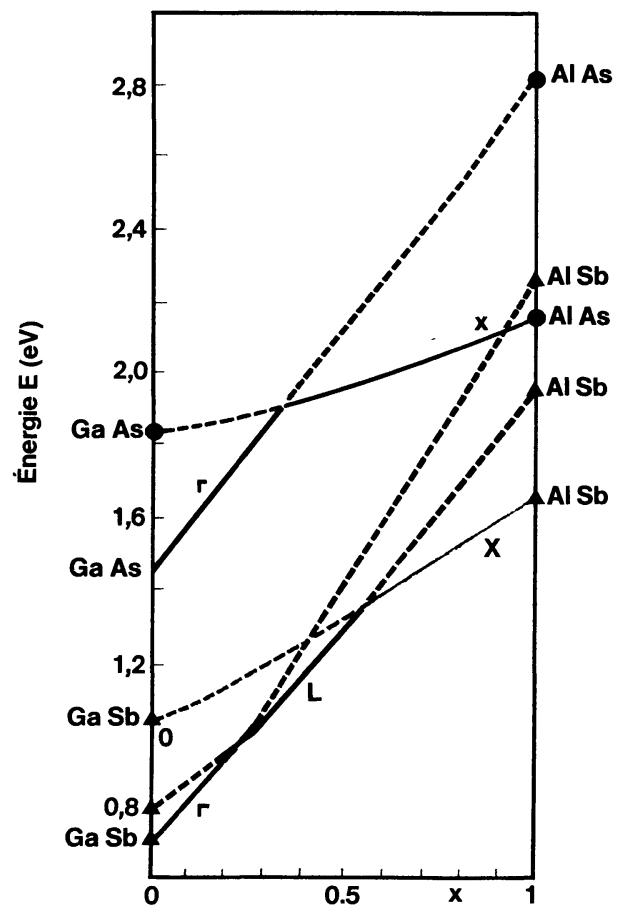

FIG. 4. - Variation des bandes interdites directes $(\Gamma)$ et indirectes $(L, X)$ avec la composition dans les alliages $\mathrm{Ga}_{1-x} \mathrm{Al}_{x} \mathrm{As}$ [25] et $\mathrm{Ga}_{1-x} \mathrm{Al}_{x} \mathrm{Sb}$ [26].

[Variation of the direct $(\Gamma)$ and indirect $(L, X)$ bandgaps in $\mathrm{Ga}_{1-x} \mathrm{Al}_{x} \mathrm{As}$ [25] and $\mathrm{Ga}_{1-x} \mathrm{Al}_{x} \mathrm{Sb}$ [26] alloys vs. composition.]

pour $\mathrm{Cu}_{2} \mathrm{~S}$ la nature de la transition n'est pas claire et l'absorption élevée dans la région transparente est due aux imperfections du cristal. 


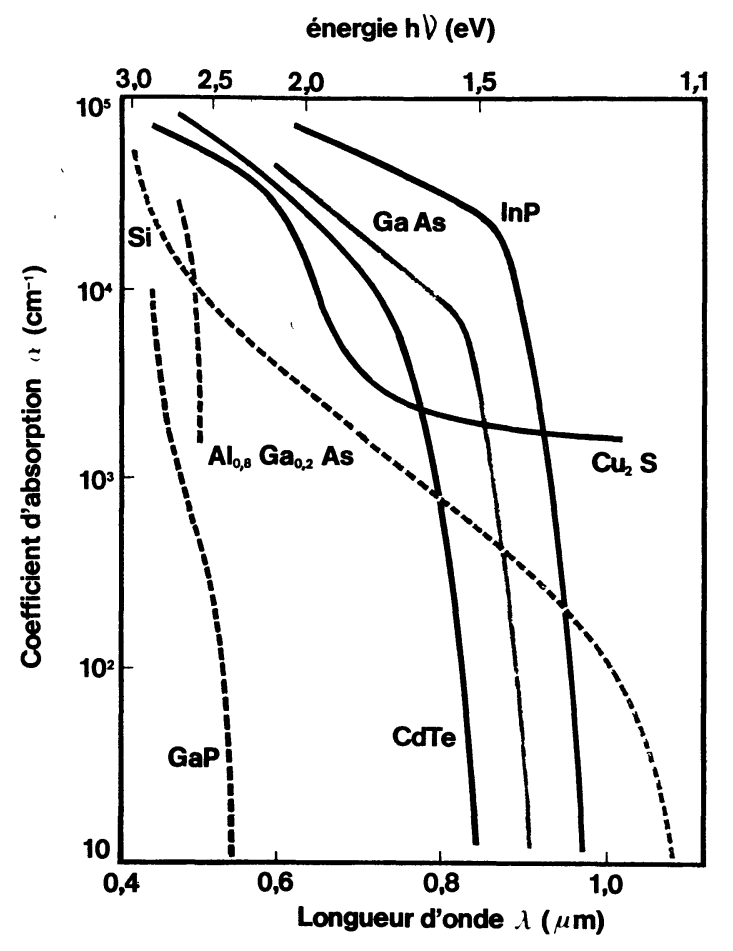

FIG. 5. - Spectre d'absorption de divers semi-conducteurs monocristallins.

[Absorption spectrum of various semiconductor single crystals.]

Le spectre d'absorption subit des modifications quand le dopage augmente : déplacement du front d'absorption et apparition d'absorption dans la région transparente. Pour GaAs ces effets sont appréciables pour des teneurs en électrons supérieures à $6 \times 10^{17} \mathrm{~cm}^{-3}$, ou des teneurs en trous supérieures à $10^{19} \mathrm{~cm}^{-3}$ [27].

Signalons l'intéressant comportement anormal du Si amorphe [28]: dans tout le domaine du visible le coefficient d'absorption est accru comme pour un matériau à transitions directes.

Les mobilités des porteurs données par le tableau IV sont, pour Si et les composés III-V et II-VI, les meilleures valeurs pour les cristaux ultrapurs; les valeurs réelles décroissent rapidement quand le dopage augmente (Tableaux V et VI). Pour les autres matériaux
TABLeAu $\mathrm{V}$

Mobilité et durée de vie électronique dans $\mathrm{GaAs}$ dopé $\mathrm{Ge}$ [29]

[Electron mobility and lifetime for $\mathrm{Ge}$ doped $\mathrm{GaAs}$ [29]]

$\begin{array}{cccl}\begin{array}{c}N_{\mathrm{A}}-N_{\mathrm{D}} \\ \left(\mathrm{cm}^{-3}\right)\end{array} & \begin{array}{c}\mu_{\mathrm{n}} \\ \left(\mathrm{cm}^{2} \cdot \mathrm{V}^{-1} \cdot \mathrm{s}^{-1}\right)\end{array} & \begin{array}{c}L_{\mathrm{n}} \\ (\mu \mathrm{m})\end{array} & \begin{array}{c}\tau_{\mathrm{n}} \\ (\mathrm{ns})\end{array} \\ 6,0 \times 10^{16} & 6 \overline{500} & -\overline{0}, 0 & 38 \\ 2,0 \times 10^{18} & 3500 & 10,5 & 12,5 \\ 1,1 \times 10^{19} & 1500 & 5,5 & 11\end{array}$

on a cité les meilleures valeurs de mobilité obtenues, pour des cristaux de pureté en général mal définie.

Une mobilité électronique élevée est bénéfique pour une photopile. On peut s'en convaincre a contrario par le cas des semi-conducteurs organiques. Ceux-ci ont été cités parfois comme des matériaux utilisables pour des photopiles, mais il n'en est rien. On a pu faire des barrières de Schottky sur la phtalocyanine à l'aluminium [31]; ce matériau a un fort coefficient d'absorption dans le visible $\left(2 \times 10^{+5} \mathrm{~cm}^{-1}\right.$ pour $0,69 \mu \mathrm{m})$ et une conduction de type $p$; les électrons ont une mobilité de $0,1 \mathrm{~cm}^{2} \mathrm{~V}^{-1} \mathrm{~s}^{-1}$ et une durée de vie de $10^{-9} \mathrm{~s}$; le rendement de cellule était de $0,01 \%$. Ce mauvais résultat, essentiellement dû à la forte densité de pièges $\left(10^{18}\right.$ à $\left.10^{19} \mathrm{~cm}^{-3}\right)$ dans ce matériau, illustre bien la nécessité d'une mobilité suffisante des porteurs de charge pour obtenir un rendement acceptable.

6. Longueurs de diffusion. - Les porteurs de charge hors d'équilibre peuvent d'abord être recombinés par des processus bande-à-bande (inverses de l'absorption). C'est le cas dans les matériaux à transitions directes comme GaAs. Pour ce dernier les données du tableau V et celles, plus générales, de la figure 6 montrent comment le taux de recombinaison croît avec le dopage.

Ce mécanisme est peu efficace dans le silicium, où la recombinaison procède essentiellement par transitions bande-niveau, c'est-à-dire inclut comme étapes intermédiaires des transitions d'électrons et de trous sur des

\section{TABLEAU VI}

Mobilités et durées de vie dans le $\mathrm{Si}$ [30]

[Carrier mobilities and lifetimes in Si [30]]

\begin{tabular}{|c|c|c|c|c|c|c|c|}
\hline $\begin{array}{c}n \\
\left(\mathrm{~cm}^{-3}\right)\end{array}$ & $\left(\mathrm{cm}^{2} \cdot \mathrm{V}^{\mu_{\mathrm{n}}} \cdot \mathrm{s}^{-1}\right)$ & $\begin{array}{c}L_{\mathrm{p}} \\
(\mu \mathrm{m})\end{array}$ & $\underset{(\mu \mathrm{p})}{\tau_{\mathrm{p}}}$ & $\begin{array}{c}P \\
\left(\mathrm{~cm}^{-3}\right)\end{array}$ & $\left(\mathrm{cm}^{2} \cdot \mathrm{V}^{\mu_{\mathrm{p}}} \cdot \mathrm{s}^{-1}\right)$ & $\begin{array}{c}L_{\mathrm{n}} \\
(\mu \mathrm{m})\end{array}$ & $\begin{array}{c}\tau_{\mathrm{n}} \\
(\mu \mathrm{s})\end{array}$ \\
\hline $10^{15}$ & 1300 & 120 & 12 & $10^{15}$ & 430 & 300 & 30 \\
\hline $10^{16}$ & 1000 & 100 & 9 & $10^{16}$ & 400 & 270 & 27 \\
\hline $10^{17}$ & 740 & 60 & 4,4 & $10^{17}$ & 220 & 200 & 18 \\
\hline $10^{18}$ & 240 & 25 & 1,2 & $10^{18}$ & 95 & 70 & 6 \\
\hline $10^{19}$ & 100 & 6 & 0,2 & $10^{19}$ & 55 & 10 & 0,6 \\
\hline $10^{20}$ & 70 & 2 & 0,2 & $10^{20}$ & 45 & 2 & 0,2 \\
\hline
\end{tabular}




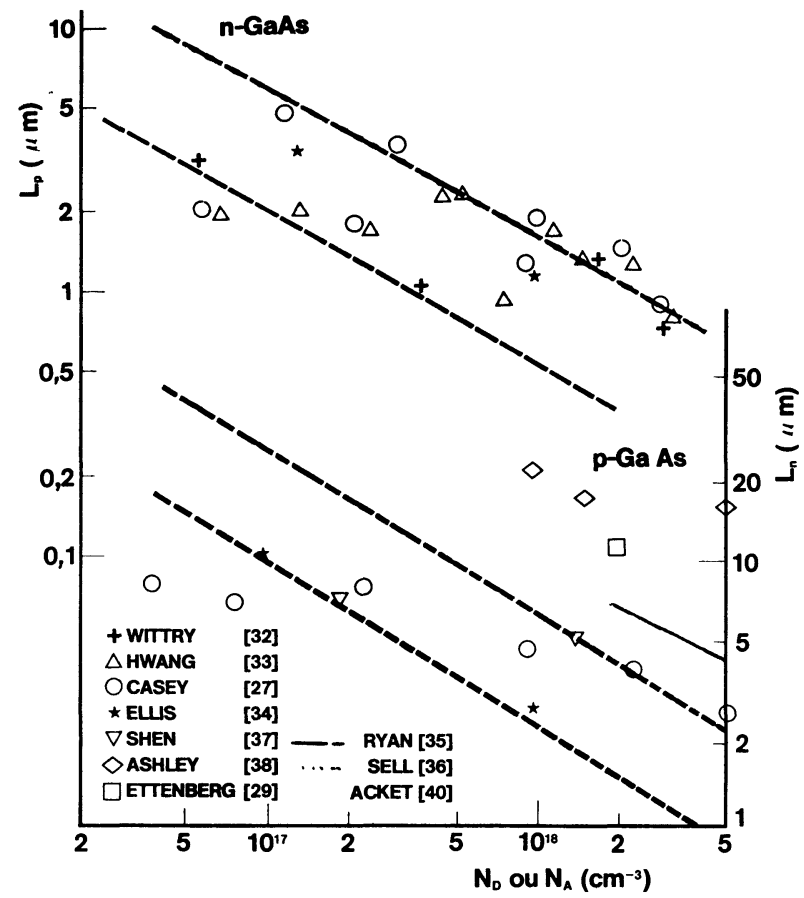

Fig. 6. - Longueur de diffusion des porteurs de charge dans $\mathrm{GaAs}$ pour différentes natures et différents niveaux de dopage. [Diffusion length of minority carriers in GaAs vs. doping level.]

niveaux d'impureté à l'intérieur de la bande interdite. Les durées de vie qui en résultent sont très élevées pour le $\mathrm{Si}$ pur mais décroissent rapidement par dopage (Tableau VI, Fig. 7). Chaque défaut dans un état de charge déterminé est caractérisé par un coefficient de capture pour les électrons $\sigma_{\mathrm{n}}$ (et pour les trous $\sigma_{\mathrm{p}}$ ). Selon la théorie usuelle, pour un matériau de type $\mathrm{p}$ ayant $N_{\mathrm{t}}\left(\mathrm{cm}^{-3}\right)$ pièges d'énergie $E_{\mathrm{t}}$ telle que

$$
E_{\mathrm{t}}-E_{\mathrm{v}}>E_{\mathrm{g}} / 2,
$$

à fort dopage la durée de vie des électrons est donnée par $1 / \tau_{\mathrm{n}}=N_{\mathrm{t}} C_{\mathrm{n}}$ (avec $C_{\mathrm{n}}=V \sigma_{\mathrm{n}}, V=$ vitesse thermique des électrons); à faible dopage les deux coefficients $C_{\mathrm{p}}$ et $C_{\mathrm{n}}$ interviennent dans $\tau_{\mathrm{n}}$, qui augmente. Pour la plupart des séries de mesures publiées, cette théorie n'est pas en bon accord avec l'expérience, pour

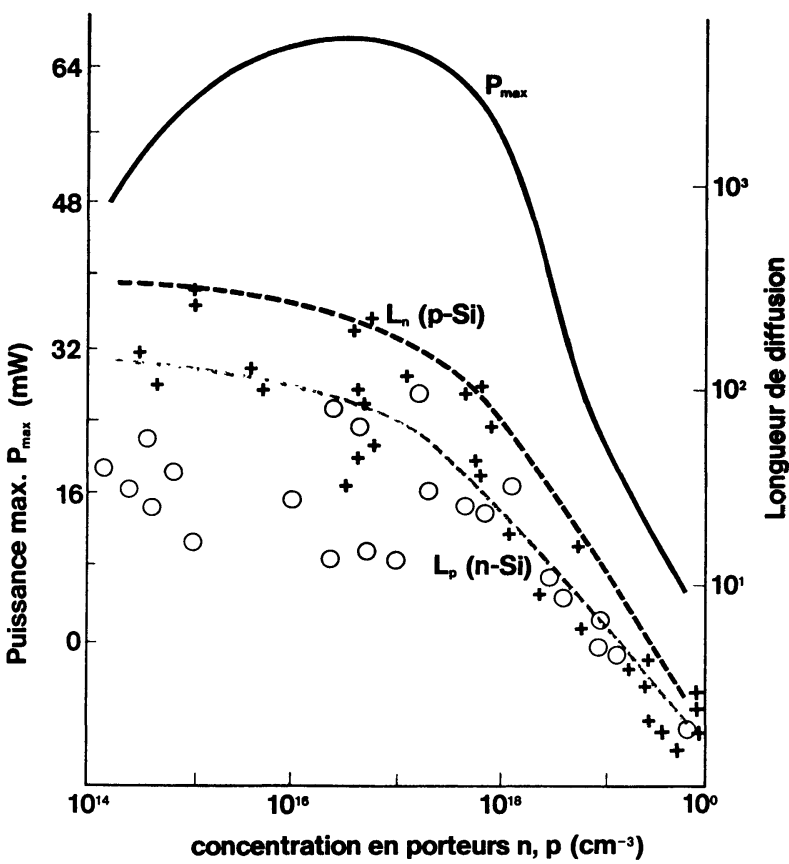

Fig. 7. - Effet du dopage sur les longueurs de diffusion mesurées dans le $\mathrm{Si}\left(\bigcirc L_{\mathrm{p}}, \triangle L_{\mathrm{n}}\right.$; les courbes suivent les valeurs maximales) et sur la puissance maximale produite à $28^{\circ} \mathrm{C}$ par des photopiles de surface $4 \mathrm{~cm}^{2}$ [30].

[Effects of doping level on the measured diffusion length in $\mathrm{Si}\left(\bigcirc L_{\mathrm{p}}, \triangle L_{\mathrm{n}}\right.$; the curves point to the maximum values) and on the calculated maximum power of $4 \mathrm{~cm}^{2} \mathrm{Si}$ cells at $28^{\circ} \mathrm{C}$ [30].]

plusieurs raisons : intervention de plusieurs types de pièges, effets d'écran qui modifient l'interaction entre porteurs libres et pièges, recombinaison Auger qui à elle seule conduit à $\tau_{n}, \tau_{\mathrm{p}} \simeq 2$ à $4 \mu \mathrm{s}$ pour $\mathrm{p}$, $n=10^{18} \mathrm{~cm}^{-3}$ [41].

La figure 7 montre également l'effet calculé du dopage sur le rendement de cellules solaires au $\mathrm{Si}$, du fait que le courant de court-circuit est corrélé à la durée de vie.

On a étudié l'influence d'impuretés spécifiques sur la durée de vie dans le $\mathrm{Si}$, par deux approches distinctes. Une approche fondamentale a permis de déterminer les sections efficaces de capture de certaines impuretés : des résultats sont donnés par le tableau VII. Une

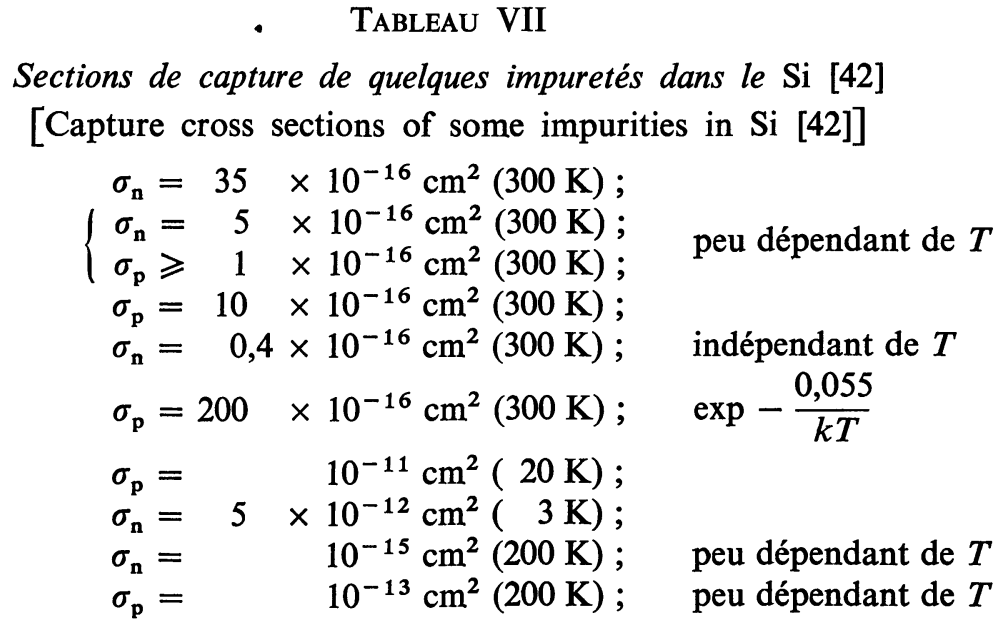

\begin{tabular}{|c|c|c|}
\hline $\mathrm{Au}^{+}$ & $\sigma_{\mathrm{n}}=35$ & $\times 10^{-16} \mathrm{~cm}^{2}(300 \mathrm{~K})$ \\
\hline $\mathrm{Au}^{0}$ & $\sigma_{\mathrm{n}}=5$ & $\times 10^{-16} \mathrm{~cm}^{2}(300 \mathrm{~K})$ \\
\hline $\mathrm{Au}^{-}$ & $\begin{array}{l}\sigma_{\mathrm{p}} \geqslant 1 \\
\sigma_{\mathrm{n}}=10\end{array}$ & $\begin{array}{l}\times 10^{-16} \mathrm{~cm}^{2}(300 \mathrm{~K}) \\
\times 10^{-16} \mathrm{~cm}^{2}(300 \mathrm{~K})\end{array}$ \\
\hline $\mathrm{In}^{0}$ & $\sigma_{\mathrm{n}}=0,4$ & $4 \times 10^{-16} \mathrm{~cm}^{2}(300 \mathrm{~K}$ \\
\hline $\mathrm{In}^{-}$ & $\sigma_{\mathrm{p}}=200$ & $\times 10^{-16} \mathrm{~cm}^{2}(300 \mathrm{~K})$ \\
\hline $3^{-}$ & $\sigma_{\mathrm{p}}=$ & $10^{-11} \mathrm{~cm}^{2}(20$ \\
\hline & $\sigma_{\mathrm{n}}=$ & $\times 10^{-12} \mathrm{~cm}^{2}(3)$ \\
\hline$n$ & $\sigma_{\mathrm{n}}=$ & $10^{-15} \mathrm{~cm}^{2}(200$ \\
\hline & & $0^{-13}$ \\
\hline
\end{tabular}


approche plus empirique a consisté à ajouter une impureté donnée à du Si ultrapur et à comparer les rendements de piles solaires construites avec tous ces matériaux, à dopage et traitement thermique constants. Le tableau II mentionne l'effet de quelques impuretés, ramenant vers $1-5 \mu$ s la durée de vie qui était de 5-20 $\mu$ s dans le $\mathrm{Si}$ ultrapur initial. Ces résultats sont confirmés en ordre de grandeur (mais non en détail) par deux études récentes [5].

Concluons, sur la recombinaison dans le $\mathrm{Si}$, que :

- pour des dopages voisins de $10^{17}$ à $10^{18} \mathrm{~cm}^{-3}$ convenant à la fabrication des photopiles, la longueur de diffusion dans les monocristaux est supérieure à la longueur d'absorption de la lumière visible, sauf si certaines impuretés critiques sont assez abondantes;

- les teneurs acceptables de chaque impureté commencent à être connues dans les monocristaux, mais l'étude des polycristaux reste à faire (rôle des joints de grain).

Dans $\mathrm{Cu}_{2} \mathrm{~S}$ et $\mathrm{CdTe}$, la situation n'est pas aussi claire que pour GaAs ou Si. Dans le premier, sous la forme de polycristal à grains fins, la longueur de diffusion des électrons serait de 0,3 à $0,8 \mu \mathrm{m}$. Pour $\mathrm{CdTe}$, le tableau VIII montre quelques résultats expérimentaux

\section{TABLEAU VIII}

Longueurs de diffusion dans $\mathrm{CdTe}$

[Diffusion lengths in CdTe]

\begin{tabular}{|c|c|c|c|}
\hline $\begin{array}{l}\text { Pureté et dopant } \\
\qquad\left(\mathrm{cm}^{-3}\right)\end{array}$ & $\begin{array}{c}\tau \\
(\mathrm{s})\end{array}$ & $\underset{(\mu \mathrm{m})}{L}$ & Réf. \\
\hline$n=5 \times 10^{14} \quad(\mathrm{In})$ & & $L_{\mathrm{p}}=1$ & [44] \\
\hline$p=10^{16}$ & & $L_{\mathrm{n}}=2,5$ & [44] \\
\hline $\begin{array}{l}\simeq 3 \times 10^{16} \text { donneurs et } \\
\text { accepteurs } \text { (compensa- } \\
\text { tion par } \mathrm{Cl} \text { ) }\end{array}$ & $\simeq 10^{-6}$ & $\simeq 10$ & [43] \\
\hline$n=6,3 \times 10^{17}$ & $\tau_{p}=6,8 \times 10^{-9}$ & $L_{\mathrm{p}}=0,82$ & [43] \\
\hline$p=1,7 \times 10^{17}(\mathrm{P})$ & $\tau_{\mathrm{n}}=10^{-10}$ & $L_{\mathrm{n}}=0,1$ & [45] \\
\hline $\begin{array}{l}n=5,5 \times 10^{17}(\text { In }) \\
\text { idem }+ \text { champ accéléra- }\end{array}$ & $\begin{array}{c}\tau_{\mathrm{p}}=5,4 \times 10^{-8} \\
L_{\mathrm{p}}(\text { apparent })=\end{array}$ & $\begin{aligned} & L_{\mathrm{p}}=3,3 \\
= & 90\end{aligned}$ & [39] \\
\hline
\end{tabular}

assez dispersés et parfois contradictoires. Des longueurs de diffusion supérieures à la longueur d'absorption sont obtenues dans CdTe compensé, mais ce matériau est trop résistant pour en faire des photopiles efficaces ; pour CdTe dopé, la longueur de diffusion est beaucoup plus faible, ce qui doit abaisser le rendement de jonctions p-n : à dopage constant de $10^{16} \mathrm{~cm}^{-3}$, et sans tenir compte de la recombinaison superficielle celui-ci serait de $27 \%$ pour $\tau \infty$, mais seulement de $20 \%$ pour $\tau=10^{-7} \mathrm{~s}$, de $10 \%$ pour $\tau=10^{-8}$ s et de $3 \%$ pour $\tau=10^{-9} \mathrm{~s}$ [43]. Ainsi les pertes par recombinaison ne sont pas négligeables dans des jonctions $\mathrm{p}-\mathrm{n}$ de CdTe. Pour éviter cette difficulté il a été proposé de créer un champ interne au voisinage de la jonction, par un gradient de $E_{\mathrm{g}}$; ce dernier est produit par un gradient de composition d'alliage.
Pour des alliages $\mathrm{Cd}_{1-x} \mathrm{Hg}_{x} \mathrm{Te}(x<0,1)$, on a montré [39] que $L_{\mathrm{p}}$ pouvait ainsi atteindre $90 \mu \mathrm{m} \mathrm{au}$ lieu de $3,3 \mu \mathrm{m}$ pour $\mathrm{CdTe}$ homogène : voir figure 8 .

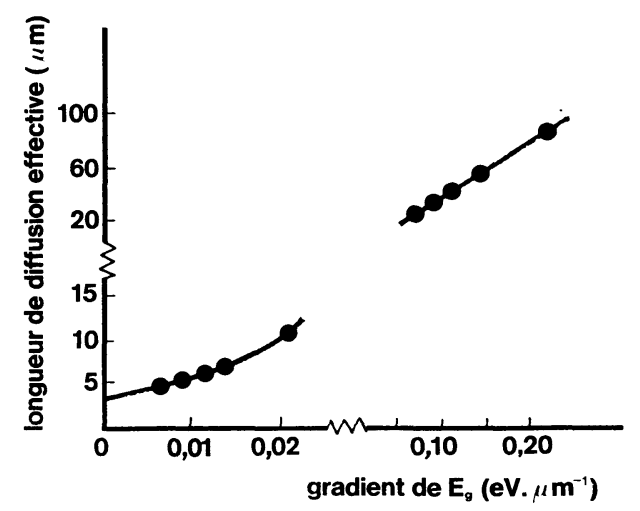

FIG. 8. - Effet d'un champ interne formé par alliage sur la longueur de diffusion effective des trous [39].

[Effect of an internal field caused by alloying on the effective hole diffusion length [39].]

7. Structures collectrices. - Le fonctionnement des jonctions $p-n$ dépend du niveau de dopage des deux régions. La différence des deux niveaux de Fermi, qui détermine la tension de circuit ouvert $V_{\text {oc }}$, croît avec le dopage. Mais on a vu qu'un fort dopage diminue le courant du circuit ouvert $J_{\text {sc à cause des pertes par }}$ recombinaison. De ce compromis résulte le dopage optimal (cf. Fig. 7). Pour une analyse plus détaillée, incluant aussi le facteur de courbe, nous renvoyons par exemple au livre de Hovel [7]. Des jonctions p-n sont également faisables avec GaAs et InP, ainsi qu'avec CdTe [45, 46].

Dans les jonctions $\mathrm{p}-\mathrm{n}$, la continuité cristalline entre les deux régions peut être parfaite, mais la surface libre est riche en défauts causant une recombinaison superficielle élevée. Cet effet est particulièrement néfaste pour les jonctions p-n de GaAs, du fait de la faible longueur d'absorption. Pour en réduire l'importance, on peut créer dans la couche superficielle un gradient de dopage [7] ou un gradient de $E_{\mathrm{g}}$ [47] ; on peut aussi supprimer l'effet des défauts de surface en déposant sur la couche superficielle une fenêtre transparente constituée par un matériau comme $\mathrm{Ga}_{x} \mathrm{Al}_{1-x} \mathrm{As}$ ou AlAs, qui s'épitaxie parfaitement sur GaAs : les piles solaires ainsi obtenues ont des rendements trés élevés [48].

Les performances de jonction $\mathrm{p}$-n sont comparées, dans le tableau IX à celles d'autres structures. Ces dernières sont étudiées pour leur intérêt particulier dans deux cas :

- celui des matériaux polycristallins, dans lesquels les traitements thermiques usuels (diffusion, épitaxie) pour former des jonctions $\mathrm{p}-\mathrm{n}$ risquent de donner des surfaces de jonction irrégulières,

- celui des nombreux composés qui n'existent que d'un seul type (n ou p), à cause de phénomènes d'autocompensation qui sont décrits par exemple par les références [49]. 


\section{TABleau IX}

Meilleur rendement de divers types de cellules solaires (sous Air Mass 1 sauf indication contraire)

[Best efficiency of various types of solar cells (at one atmosphere unless indicated)]

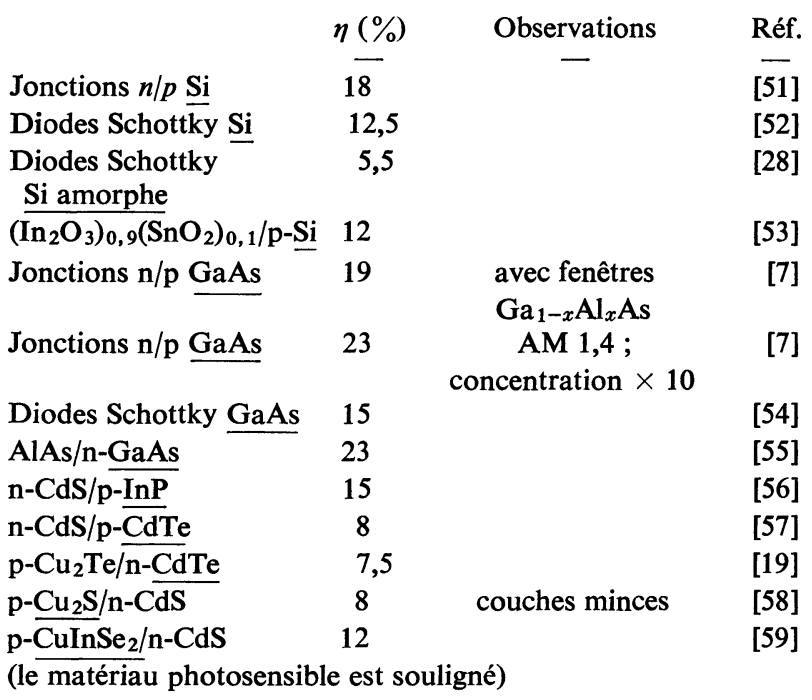

Des diodes Schottky (semi-conducteur-métal) efficaces peuvent être construites sur $\mathrm{Si}$ ou GaAs (cf. Tableau IX). On a pu par exemple obtenir $\eta=9 \%$ sur Si sans couche antiréflectrice sans porter la cellule au-dessus de la température ambiante ; les caractéristiques de ces cellules sont très sensibles aux états d'interface ; on a trouvé qu'une mince couche d'oxyde à l'interface est bénéfique [50].

Les performances des hétérojonctions dépendent surtout de deux propriétés d'interface :

- les discontinuités de la structure de bande (Fig. 9), déterminées par les valeurs relatives des largeurs de bande interdite $E_{\mathrm{g} 1}, E_{\mathrm{g} 2}$ et des affinités électroniques des deux matériaux (cf. Tableau 4). Ces discontinuités :

$$
E_{\mathrm{c}}=\chi_{1}-\chi_{2}, \quad E_{\mathrm{v}}=\left(\chi_{1}-\chi_{2}\right)+\left(E_{\mathrm{g} 1}-E_{\mathrm{g} 2}\right)
$$

commandent la nature des courants direct et inverse à travers la structure (cf. modèles théoriques dans les réf. [7] et [24]);

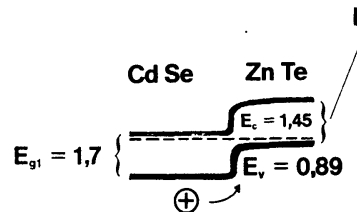

(a)

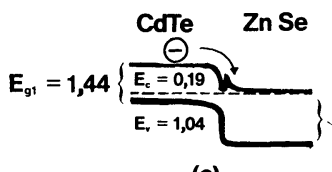

(c)$$
E_{92}=2,26
$$

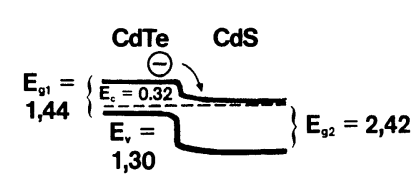

(b)

Fig. 9. - Schéma de bandes de quatre hétérojonctions.

[Band scheme of four heterojunctions.]
- la différence de paramètre de réseau des deux matériaux : la croissance épitaxique d'un matériau sur un autre est possible si leurs paramètres diffèrent de moins de $10 \%$, mais de nombreux défauts sont générés s'ils diffèrent de plus de $1 \%$. Quand ces jonctions sont refroidies à partir de températures élevées, la différence des coefficients de dilatation est également importante (Tableau IV).

La formation d'hétérojonctions, et aussi celle de fenêtres transparentes, peut être discutée par référence à la figure 10. Les performances remarquables de

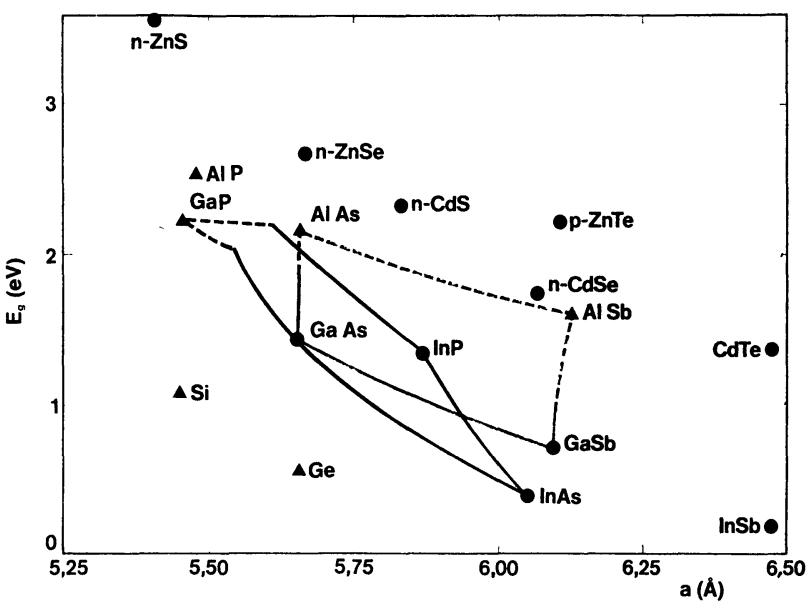

Fig. 10. - Paramètre de réseau et largeur de bande interdite des principaux matériaux pour photopiles $(\Theta,-$ transitions directes ; $\mathbf{\Delta}, \ldots$ transitions indirectes).

[Lattice parameters and bandgaps of solar cell material candidates $(\boldsymbol{O}, \ldots$ direct transitions ; $\mathbf{\Lambda},-$ - - indirect transitions).]

$\mathrm{AlAs} / \mathrm{GaAs}$ et de $\mathrm{CdS} / \mathrm{InP}$ correspondent à des cas d'excellent accord paramétrique. Mais par contre le grand désaccord paramétrique de $\mathrm{CdS}$ et de $\mathrm{CdTe}$ n'empêche pas la formation d'hétérojonctions assez bonnes. Aussi l'étude détaillée des interfaces d'hétérojonctions peut seule permettre de comprendre chaque cas particulier; il faudrait caractériser les profils de composition et les profils de dopage, déterminer les états-pièges à l'interface et mesurer le taux de recombinaison propre à l'interface. Même dans le cas de la figure $9 d$ qui est étudiée depuis de nombreuses années, ceci n'a pas encore été fait complètement.

8. Fiabilité des piles solaires. - Les piles solaires sont normalement encapsulées (de préférence par du verre) pour les protéger de l'humidité. Dans ces conditions la durée de vie des cellules au $\mathrm{Si}$ est au moins de dix ans. Cette fiabilité est encore insuffisante ; les calculs du paragraphe 2 supposaient une durée de vie de 20 ans. De nombreux programmes d'études technologiques sont en cours sur ce point. Nous développerons plus particulièrement ce problème dans le cas de photopiles au $\mathrm{Cu}_{2} \mathrm{~S} / \mathrm{CdS}$ qui étaient considérées, encore récemment, comme peu fiables, mais ont fait l'objet d'une étude récente [60] qui les a améliorées de façon décisive sur ce point. 
Des modifications réversibles de leurs caractéristiques sous illumination ou polarisation ont été expliquées par la présence de pièges à l'interface : si des électrons injectés de $\mathrm{Cu}_{2} \mathrm{~S}$ dans CdS sont capturés par ces pièges, la hauteur de barrière, et donc aussi $V_{\text {oc }}$, sont modifiées. Il se trouve que cet effet n'a pas lieu si CdS est fortement dopé. Mais il reste alors une autre cause d'évolution.

$\mathrm{Si}$ la couche de $\mathrm{Cu}_{2} \mathrm{~S}$ subit une décomposition chimique et si des ions $\mathrm{Cu}^{+}$diffusent dans $\mathrm{CdS}$, alors se produisent des courts-circuits localisés, causant une chute irréversible du rendement de la cellule. Dans le diagramme de phases $\mathrm{Cu}-\mathrm{S}$, il existe une phase orthorhombique qui se présente à $300 \mathrm{~K}$ sous deux structures similaires : la chalcocite $\left(\mathrm{Cu}_{2,00} \mathrm{~S}\right)$ et la djurléite $\left(\mathrm{Cu}_{1,96} \mathrm{~S}\right)$. La chalcocite peut être oxydée en djurléite selon la réaction

$$
\mathrm{Cu}_{2} \mathrm{~S} \rightleftharpoons 0,04 \mathrm{Cu}+\mathrm{Cu}_{1,96} \mathrm{~S} .
$$

La figure 11 montre la variation d'énergie libre avec l'indice $x$ du $\mathrm{Cu}_{x} \mathrm{~S}$ (exprimée en volts : $E_{0}$ est un poten-

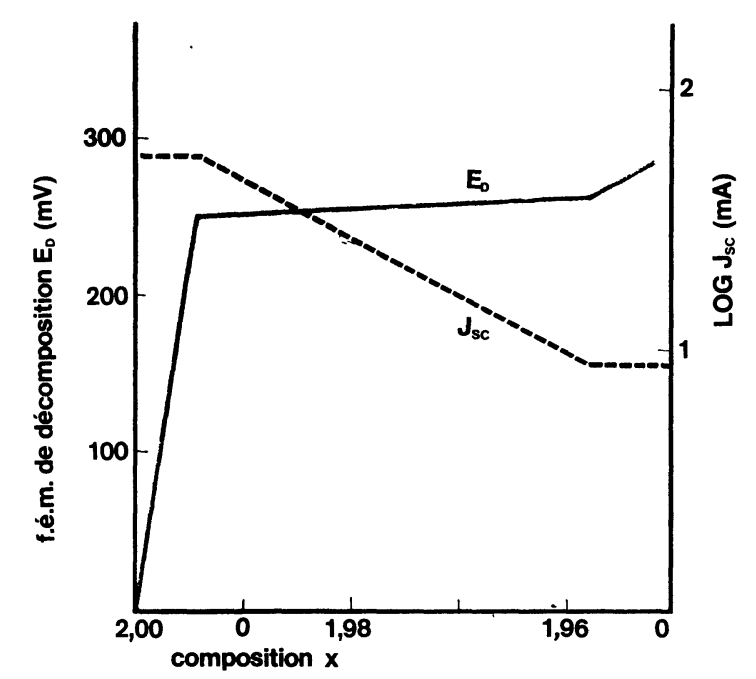

FIG. 11. - Effet de la composition $x$ de $\mathrm{Cu}_{x} \mathrm{~S}$ sur la fém. de décomposition de ce matériau et sur le courant de court-circuit $J_{\text {sc }}$ de cellules $\mathrm{Cu}_{2} \mathrm{~S} / \mathrm{CdS}$.

[Effect of the composition $x$ of $\mathrm{Cu}_{x} \mathrm{~S}$ on the decomposition e.m.f. of this material and on the short-circuit current $J_{\mathrm{sc}}$ of $\mathrm{Cu}_{x} \mathrm{~S} / \mathrm{CdS}$ cells.]

tiel de décomposition) : la réaction ci-dessus est tenue d'avoir lieu si la couche de $\mathrm{Cu}_{x} \mathrm{~S}$ est soumise à une f. é. m. supérieure à $E_{0}$. Il est donc avantageux de choisir une composition $x \neq 2$ conduisant à une valeur élevée de $E_{0}$; comme le courant de court-circuit, de son côté, varie peu avec $x$, la composition optimale est celle de la limite du domaine d'existence de la chalcocite : $x=1,995$. Cette composition peut être ajustée par une technique électrochimique.

De plus la réaction ci-dessous est accélérée si le $\mathrm{Cu}$ résultant diffuse dans la couche de $\mathrm{CdS}$, diffusion qui est assistée par le champ électrique présent lorsque la cellule fonctionne. On peut diminuer cette diffusion en formant un polycristal de CdS à gros grains et en ajoutant des impuretés comme $\mathrm{Al}$ qui forme des complexes $\left(V_{\mathrm{Cd}} \mathrm{Al}_{\mathrm{Cd}}\right)$ diminuant la teneur en lacunes (la diffusion a lieu par un mécanisme lacunaire).

En ajustant ainsi la composition de $\mathrm{Cu}_{x} \mathrm{~S}$ et de $\mathrm{CdS}$, on a pu obtenir des cellules très fiables.

9. Aptitude des piles solaires à être utilisées sous concentration. - Utiliser une photopile sous rayonnement solaire concentré oblige à en modifier la conception de plusieurs façons. Si le rapport de concentration $K$ est élevé, des précautions devront être prises pour éviter un échauffement (contacts thermiques, refroidissement forcé). De plus le courant de court-circuit $J_{\text {sc }}$ croissant linéairement avec $K$, les pertes par résistancesérie deviennent prohibitives si on ne choisit pas un matériau plus dopé et une grille de contact sur la couche superficielle plus serrée. L'accroissement du dopage optimal est important : pour du Si de base de type $\mathrm{p}$, il passe de $10^{15} \mathrm{~cm}^{-3}$ pour $K=1$ à $10^{17} \mathrm{~cm}^{-3}$ pour $K=1000$ selon [61]. Pour plus de détails sur ces problèmes, nous renvoyons aux références [62].

Le silicium semble permettre, sans difficulté, d'atteindre des rapports de concentration de quelques dizaines. Mais on doit souligner l'intérêt que présenterait une valeur de $K$ de l'ordre de 1000 : une concentration précise n'est pas beaucoup plus chère qu'une concentration sommaire et permettrait d'utiliser des photopiles de prix au $\mathrm{m}^{2}$ beaucoup plus élevé que la simple exposition directe. Il semble acquis que les photopiles à $\mathrm{GaAs}$ sont capables de fonctionner sous haute concentration. James et Moon [48] ont mesuré un rendement de $17,5 \%$ pour $K=312$. De plus, grâce à sa valeur élevée de $E_{\mathrm{g}}$, GaAs supporte également une certaine élévation de température : $\eta$ reste de $14 \%$ à $200^{\circ} \mathrm{C}$ sous la même concentration. On peut imaginer, à partir de ce résultat, la production simultanée d'électricité et d'eau chaude.

10. Conclusions. - Dans le tableau $X$, nous avons schématisé les caractéristiques principales des quatre filières qui semblent aujourd'hui les plus capables de succéder à la technologie actuelle (qui peut elle-même être améliorée notablement) ( $\S 4$, cas $a$ ) et par conséquent subsister encore quelques années.

Sans concentration, on peut envisager une production de panneaux de cellules au Si polycristallin coûtant environ $1000 \mathrm{~F} / \mathrm{m}^{2}$ (soit dix fois moins qu'en 1976). Un des scénarios possibles est le suivant : à partir de matériau purifié de façon relativement sommaire, le $\mathrm{Si}$ sera produit par exemple par trempage et la technologie de fabrication de la photopile sera entièrement automatisée. Un rendement de $12 \%$ est vraisemblable dans ces conditions, d'ici une dizaine d'années. Le coût total du générateur compte une partie fixe, indépendante du type de photopiles, constituée par le coût de la structure porteuse, du conditionnement et de la régulation d'énergie, que nous estimons à $500 \mathrm{~F} / \mathrm{m}^{2}$. Un coût de l'énergie de $1 \mathrm{~F} / \mathrm{kWh}$ sera alors accessible. 


\section{TABleaU $X$}

Caractéristiques prévisibles à long terme des principales filières

[Main characteristics of the best future materials]

\begin{tabular}{|c|c|c|c|c|}
\hline \multirow[b]{2}{*}{ Matériau photosensible } & \multicolumn{2}{|c|}{ Si polycristal } & \multirow{2}{*}{$\begin{array}{l}\mathrm{CdTe} \mathrm{ou} \mathrm{Cu}_{2} \mathrm{~S} \\
\text { polycristal } \\
\text { sans } \\
\text { concentration }\end{array}$} & \multirow{2}{*}{$\begin{array}{c}\text { GaAs } \\
\text { polycristal } \\
\text { sous } \\
\text { concentration }\end{array}$} \\
\hline & $\begin{array}{l}\text { sans } \\
\text { concentration }\end{array}$ & $\begin{array}{c}\text { sous } \\
\text { concentration }\end{array}$ & & \\
\hline Longueur d'absorption $\quad(\mu \mathrm{m})$ & \multicolumn{2}{|c|}{50} & 1 & 2 \\
\hline $\begin{array}{l}\text { Longueur de diffusion au }(\mu \mathrm{m}) \\
\text { dopage optimal }\end{array}$ & \multicolumn{2}{|c|}{100} & \multicolumn{2}{|c|}{2 (matériau homogène) } \\
\hline \multirow[t]{2}{*}{ Epaisseur optimale } & \multirow{2}{*}{\multicolumn{2}{|c|}{$\begin{array}{l}50 \\
50\end{array}$}} & \multicolumn{2}{|c|}{20 (alliage à $E_{\mathrm{g}}$ variable) } \\
\hline & & & 5 & 5 \\
\hline \multirow{3}{*}{$\begin{array}{l}\text { Procédé d'élaboration suppo } \\
\text { le plus économique } \\
\text { Objectif de rendement }(\%)\end{array}$} & \multicolumn{2}{|c|}{$\begin{array}{l}\text { trempage ou moulage ? } \\
\text { (Si « métallurgique » pur) }\end{array}$} & pulvérisation & $\begin{array}{l}\text { dépôt chimique } \\
\text { en phase vapeur }\end{array}$ \\
\hline & & & & \\
\hline & 12 & 10 & 10 & 12 \\
\hline Rapport de concentration $K$ & 1 & 30 & 1 & 1000 \\
\hline $\begin{array}{l}\text { Objectif de coût }\left(\mathrm{F} / \mathrm{m}^{2}\right) \text { de collec- } \\
\text { teur solaire }\end{array}$ & 1500 & 1000 & 1000 & 1200 \\
\hline Objectif de coût $(\mathrm{F} / \mathrm{kWh})$ & 1 & 0,8 & 0,7 & 0,8 \\
\hline
\end{tabular}

Une alternative est constituée par la pile au $\mathrm{Cu}_{2} \mathrm{~S}$ ou CdTe supposée produite par un procédé entièrement chimique.

Les filières sous concentration impliquent la présence d'héliostats coûtant 300 à $600 \mathrm{~F} / \mathrm{m}^{2}$ (en supposant une fabrication de grande série). La surface de cellule étant divisée par $K$, le prix des cellules devient un élément mineur du bilan ; c'est pourquoi il serait concevable, au cas où les techniques de préparation mentionnées au tableau $X$ se révéleraient inacceptables (instabilité des polycristaux sous forte illumination par exemple), de revenir au matériau monocristallin sans modifier beaucoup le coût global.

On peut montrer aisément qu'aucune de ces filières ne conduit à un risque de raréfaction des matières premières. Pour installer en 20 ans une puissance totale satisfaisant $1 \%$ des besoins de la société, il faudrait consommer annuellement 50000 tonnes de Si (ce qui représente cependant 25 fois la quantité nécessaire à la microélectronique), or les ressources en $\mathrm{Si}$ sont illimitées. Pour le CdTe, la consommation annuelle de $\mathrm{Cd}$ serait de 5000 tonnes, soit 4 fois moins que la production actuelle et 200 fois moins que les réserves estimées. L'utilisation de la concentration rendrait ce problème encore moins critique; toutefois la filière au GaAs sous concentration impliquerait une modification par plusieurs dizaines de la production actuelle de $\mathrm{Ga}$, ce que les réserves connues permettent très amplement.

On peut chercher à comparer les systèmes photovoltaïques aux systèmes thermodynamiques de conversion de l'énergie solaire. Ces derniers ont des prix d'installation assez bien connus : 100 à $200000 \mathrm{~F} / \mathrm{kW}$ pour les collecteurs sans concentration, 20 à $30000 \mathrm{~F} / \mathrm{kW}$ pour les centrales à tour. Les photopiles d'aujourd'hui, sans et avec concentration, conduisent à des coûts comparables. Mais le stockage électrique étant beaucoup plus difficile que le stockage thermique, de grosses installations photovoltaïques sont impensables alors que les centrales thermodyna. miques à tour sont très faisables au niveau de quelques mégawatts. A part cette limitation qui peut jouer un rôle négatif sur l'avenir des photopiles, l'issue de la compétition n'est pas évidente et dépendra beaucoup du niveau atteint par la production (de photopiles d'héliostats) et son degré d'automatisation, qui peuvent causer un abaissement net, dans dix ans, des coûts indiqués ci-dessus. Rappelons que les centrales électriques classiques coûtent environ $4000 \mathrm{~F} / \mathrm{kW}$, à quoi s'ajoute le prix du combustible consommé.

Alors que plusieurs filières prometteuses se présentent, on devrait voir maintenant se développer des recherches actives sur les photopiles, en particulier sous l'angle de la science des matériaux.

\section{Bibliographie}

[1] Loferski, J. J., J. Appl. Phys., 27 (1956) 777 ; Proc.. IEEE 51 (1963) 667.

[2] DuRAND, H., Onde électrique 55 (1975) 161.

[3] Hunt, L. P., Proc. 12th IEEE Photovoltaic Spec. Conf., Baton Rouge (1976).
[4] Wakefield, G. F., Maycock, P. D., Chu, T. L., Proc. 11th IEEE Photovoltaic Spec. Conf., Phonix (1975).

[5] Davis, J. R., RaI-Choudhury, P., Blais, P. O., Hopkins, R. H., McCormicK, J. R., Proc. 12th IEEE Photovoltaic Spec. Conf., Baton Rouge (1976) ; 
Hill, D., Gutsche, H., Wang, M. S., Gupta, K. P., TUCKer, W. F., Crepin, R., ibid.

[6] Hunt, L. P., Dosaj, V. D., McCormick, J. R., Crossman, L. D., ibid.

[7] Hovel, H. J., Solar cells, in Semiconductors and Semimetals, vol. 11 (ed. Willardson, R. K., BeER, A. C. (Acad. Press) 1975).

[8] Lindmayer, J., Proc. 12th IEEE Photovoltaic Spec. Conf., Baton Rouge (1976) ;

FisCHER, H., PSCHUNDER, W., ibid.

[9] BRIssot, J. J., Comm. personnelle (1964) ;

HAIGH, A. D., Intern. Coll. on solar electricity, Toulouse (1976).

[10] Wolf, M., McDiarmid, A. G., Noel, G. T., Proc. 12th IEEE Photovoltaic Spec. Conf., Baton Rouge (1976).

[11] Browning, M. F., Blocher, J. M., Wilson, W. J., CarMICHAEL, D. C., ibid.

[12] Faust, J. W. Jr, Hilborn, R. B. Jr, ibid.

[13] Ravi, K. V., Gonsiorawski, R., Wald, F. V., Hari RaO, C. V., Ho, J. C. T., ibid.

[14] Heaps, J. D., ibid.

[15] BRissot, J. J., Belouet, C., Intern. Coll. on solar electricity, Toulouse (1976)

[16] Chu, T. L., Chu, S., Duh, K. V., Mollenkopf, H. C., Yoo, H. I., Proc. 12th IEEE. Photovoltaic Spec. Conf., Baton Rouge (1976).

[17] Cohen, V., Huggins, R. A., J. Electrochem. Soc. 123 (1976) 381.

[18] JoRdan, J. F., Proc. Inter. Conf. on photovoltaic power generation, Hamburg (1974)

[19] Cusano, D. A., Solid State Electr. 6 (1967) 217 ; Revue Phys. Appl. 1 (1966) 195.

[20] Coste, G., Fremy, J., Nguyen, D. T., Proc. Int. Conf. on solar cells, Toulouse (1970).

[21] Cohen-Solal, G., Sella, C., Imhoff, V., Zozime, A., Japan J. Appl. Phys. suppl. 2, part 1 (1974) 517.

[22] Van Vechten, J. A., Phys. Rev. 187 (1969) 1007.

[23] Bernard, M., Compt. Rend. Conf. Intern. sur les composés ternaires, Strasbourg 1975.

[24] Milnes, A. G., Feucht, D. L., Hétérojonctions et jonctions métal semi-conducteur (Acad. Press) 1972.

[25] Monemar, B., Shih, K. K., Pettit, G. D., J. Appl. Phys. 47 (1976) 2604.

[26] Keh-Yung Chieng, Tech. Report 5111-5 (Stanford Univ. 1975).

[27] Casey, H. C. Jr, Sell, D. D., Wecht, K. W., J. Appl. Phys. 6 (1975) 250.

[28] Carlson, D. E., Wronski, C. P., Triano, A. R., Daniel, R. E., Proc. 12th IEEE Photovolt. Spec. Conf. Bâton Rouge (1976)

[29] Ettenberg, M., Kressel, H., Gilbert, S. L., J. Appl. Phys. 44 (1973) 827

[30] Iles, P. A., Soclof, S. I., Proc. 11th IEEE Photovoltaic Spec. Conf. Phœnix (1975).

[31] Ghosh, A. K., Morel, U. L., Feng, T., Shaw, R. T., Rowe, C. A., J. Appl. Phys. 45 (1974) 230.

[32] Wittry, D. B., Kyser, D. F., J. Phys. Soc. Japan 21 (1966) 312.

[33] Hwang, C. J., J. Appl. Phys. 40 (1969) 3731.

[34] Ellis, B., Moss, T. S., Solid State Electr. B (1970) 1.

[35] Ryan, R. D., EBERHARDT, J. E., Solid-State Electr. 15 (1972) 865.

[36] Sell, D. D., Casey, H. C. Jr, J. Appl. Phys. 45 (1974) 800.
[37] SHEN, L. C. C., Tech. Report 5124-2 (Standford Union) 1976.

[38] Ashley, K. L., Carr, D. L., Romano-Moran, R., Appl. Phys. lett. 22 (1973) 23.

[39] Bouazzi, A., Mimila Arroyo, J., Proc. Int. Coll. Solar Electricity, Toulouse (1976).

[40] Acket, G. A., Nijman W., Lam, H.'t, J. Appl. Phys. 45 (1974) 3033.

[41] Beck, J. D., Conradt, R., Solid State Comm. 13 (1973) 93.

[42] Bonch-Bruevich, V. L., LandSberg, E. G., Phys. Stat. Sol. 29 (1968) 9.

[43] Bell, R. O., Serreze, H. B., Walo, F. V., Proc. 11 th IEEE Photovoltaic Spec. Conf. Phœnix (1975).

[44] Selezneva, M. A., Kuprivanovna, T. A., Ditsman, S. A., Izv. Akad Nauk SSSR, Sev. Fiz. 38 (1974) 2328.

[45] Gu, J., Kitakara, T., Fujita, S., SAKaguchi, T., Japan J. Appl. Phys. (1975) 499.

[46] Bernard, J., Lancon, R., Paparoditis, C., Rodot, M., Revue Phys. Appl. 1 (1966) 211.

[47] Konagai, M., Takahashi, K., J. Appl. Phys. 46 (1975) 3542. Hutchiy, J. A., Fudurich, R. L., J. Appl. Phys. 47 (1976) 3140 et 3152 .

[48] Woodall, J. M., Hovel, H. J., Appl. Phys. lett. 21 (1972) 379.

Hovel, H. J., Woodall, J. M., Appl. Phys. lett. 27 (1975) 447 ;

James, L. W., Moon R. L., Appl. Phys. lett. 26 (1975) 467.

[49] Mandel, G., Phys. Rev. 134 (1964) 1073 ; Mandel, G., Moreheao, F. F., Wagner, R. R., Phys. Rev. A136 (1964) 826 ; KROGER, F. A., The chemistry of imperfect crystals (North Holland) 1964.

[50] Ponpon, J. P., Siffert, P., Proc. 11th IEEE Photovoltaic Spec. Conf. Baton Rouge (1976).

[51] Lindmayer, J., Allison, J. F., Proc. 9th IEEE Photovoltaic Spec. Conf. (Silver Springs) (1972).

[52] Fabre, E., Michel, J., BAUdeT, Y., Proc. 12th IEEE Photovoltaic Spec. Conf. Baton Rouge (1976)

[53] Du Bow, J. B., Burk, D. E., Sites, J. R., Appl. Phys. lett. 29 (1976) 494.

[54] Stirn, M. J., Yeh, Y. C. M., Appl. Phys. Lett. 27 (1975) 95.

[55] Johnston, W. D., Callahan, W. M. Jr, Proc. 12th IEEE Photovoltaic Spec. Conf. Bâton Rouge (1976).

[56] Shay, J. J., Wagner, S., Bachman, K. J., Biehler, E., BETTINI, M., ibid.

[57] Fahrenbruch, A. L., Buch, F., Mitchell, K., Bube, R., ibid.

[58] Coste, G., Fremy, J., Nguyen, D. T., Proc. Int. Conf. on solar cells, Toulouse (1970).

[59] Wagner, S., Shay, L., Meliorato, P., Kasper, H. M., Appl. Phys. lett. 27 (1975) 89.

[60] Besson, J., Nguyen-Duy, J., Gauthier, A., Palz, W., Martin, C., Proc. 11th IEEE Photovoltaic Spec. Conf. Phœnix (1975).

[61] Evdokimov, V. M., Milovanov, A. F., Int. Conf. on sun at the service of mankind, Paris (1973) Photovolt, power section, p. 79.

[62] Schuelfr, D. G., Fossum, J. G., Burgets, E. L., Proc. 11th IEEE Photovoltaic Spec. Conf., Phœnix (1975) ; J. KREBS, Proc. Int. Conf. on Solar Electricity, Toulouse (1976).

[63] Carlson, D. E., Wronski, C. R., Appl. Phys. lett. 28 (1976) 671.

[64] $* * *$, Physics today, janv. 1977, p. 17 